\title{
Waves, conservation laws and symmetries of a third-order nonlinear evolution equation
}

\author{
A. Huber ${ }^{1}$ \\ ${ }^{1}$ Address constantly: Prottesweg 2a, A-8062 Kumberg, AUSTRIA
}

\begin{abstract}
In this paper a less studied nonlinear partial differential equation of the third-order is under consideration. Important properties concerning advanced character such like conservation laws and the equation of continuity are given. Characteristic wave properties such like dispersion relations and both the group and phase velocities are derived explicitly. In addition, we discuss the non-classical case relating to potential symmetries for the first time. Further, for practical applications in several domains of sciences we discuss in detail approximate symmetries. Finally, as a further new contribution we deduce new generalized symmetries of lower order. Some important notes relating to future intensions are given.
\end{abstract}

Key Words: Nonlinear partial differential equations, evolution equations, symmetries.

PACS-Code: 02.30Jr, 02.20Qs, 02.30Hq, 03.40Kf.

AMS-Classification: 35K55, 35D35.

\section{Introduction, outline the problem}

Progresses in recent years in the study and analysis of nPDEs have made significant contributions to the understanding of many physical systems. Modelling of physical systems often leads to nonlinear evolution equations of the general form $u_{t}=K[u]$, where $K[u]$ is a locally defined function (or a nonlinear operator in general) of the function $u$ and its $x$-derivatives.

Well-known evolution equations describing physical phenomena could found in several domains of applied sciences. We restrict the pool of equations to 'classical' nPDEs, such like the Korteweg de Vries Equation (Drazin and Johnson, 1989; Witham, 1974; Eilenberger, 1983) and its varieties, the cylindrical KdV (Drazin and Johnson, 1989) and the generalized KdV (Eilenberger, 1983) modeling the propagation of weakly nonlinear waves in dispersive media. Otherwise a well known variety of the KdV is known for a long time, the so-called modified KdV equation (Ablowitz and Clakson, 1991; Dodd et al., 1988), describing nonlinear acoustic waves in anharmonic lattices (Zabusty, 1967 and Alfvén waves in a collisionless plasma (Kakutani, 1969).

Here we concentrate our intensions to a less studied unnamed variety of the mKdV Equation (Fung and Au, 1984; Au and Fung, 1984), which differs from the $\mathrm{mKdV}$ Equation by a first local-derivative term whereby this term changes basically the equation's property:

$$
\frac{\partial u}{\partial t}+\frac{\partial^{3} u}{\partial x^{3}}-6 u^{2} \frac{\partial u}{\partial x}+6 \lambda \frac{\partial u}{\partial x}=0
$$

with $u=u(x, t), u \in C^{3}(-\infty, \infty),\left\{u, u_{x}, u_{t}\right\} \neq 0,(x, t) \in R, t \in R^{+}, t>0$ and $\lambda$ is a non-vanishing parameter.

We assume that the function $u(x, t)$ acts as the amplitude and is suitable therefore to describe wave propagation depending upon time $t$ in the sense of an evolution equation in which the steepening effect of the nonlinear term is counterbalanced by the (linear) dispersion term(s). 


\section{Physical properties concerning wave motion}

Up till now no direct physical applications are known and this is the crucial purpose of study in this paper. In the following without any loss of generality we can set the parameter $\lambda=1$.

Normally, the addition of an odd derivative term leads to the fact that the dispersion relation is real for real $k$, the wave vector. To see this, we assume the linear eq.(1) and introduce the 'ansatz' $u(x, t) \propto A \exp [i(\omega t-k x)]$ into eq.(1) to derive the dispersion relation $\omega=\omega(k)=k\left(6-k^{2}\right)$ for $A=1$.

This relation differs from the $\mathrm{mKdV}$ that is $\omega=\omega(k)=k^{3}$. We observe that a linear part of the wave vector is overlaid. Further, we deduce two characteristic velocities: The phase velocity $c_{p}$ and the group velocity $c_{g}$ respectively by:

$$
c_{p}=\omega / k=\left(6-k^{2}\right), \quad c_{g}=d \omega / d k=-2 k .
$$

$c_{g}$ remains negative for all $k \in R^{+}$and takes positive for all $k \in R^{-}$.

Both velocities tends to $\left(c_{p}, c_{g}\right) \rightarrow \infty$ as $k \rightarrow \infty$. That means that all waves of large wave numbers (small wavelengths) propagate in the negative $x$-direction for all $k \in R^{+}$; if they exist anyway (similar to the KdV equation). Introducing a velocity field $v(x)$ and the amplitude field $u(x)$ we deduce the equation of continuity

$$
u_{t}+(v u)_{x}=0, \quad v(u)=\frac{u_{x x}}{u}+2\left(u^{2}-3\right)
$$

where $u(x)$ and $v(x)$ are sufficiently smooth functions. This equation can be linearized for small perturbations about the equilibrium state $u=u_{0}$ and $v=v_{0}$ so that we can introduce $u=u_{0}+\widetilde{u}(x, t)$ and $v=v_{0}+\widetilde{v}(x, t)$.

Then the continuity equation (2.2) reduces to a first-order equation $\widetilde{u}_{t}+v_{0} \widetilde{u}_{x}=0$ with solutions $\tilde{u}=f\left(x-v_{0} t\right)$ representing traveling waves.

Theorem I: A general equation of the form

$$
\frac{\partial T}{\partial t}+\frac{\partial X}{\partial x}=0
$$

is called a conservation law where $T$ and $X$ are known as the density and the flux.

If both $T$ and $X$ are integrable on $(-\infty, \infty)$ so that $X$ tends to a constant as $|x| \rightarrow \infty$, then $(2.3)$ can be integrated to

$$
\frac{d}{d t}\left[\int_{-\infty}^{\infty} T d x\right]=0 \quad \text { or equivalently } \int_{-\infty}^{\infty} T d x=\text { const. }
$$

where the latter integral is called a constant of motion. This leads to the following

\section{Theorem II:}

The nPDE (1) admits three constants of motion: (i) the conservation of mass, (ii) the conservation of the horizontal momentum and (iii) the conservation of energy so that

$$
\begin{aligned}
& \frac{\partial}{\partial t} u=\frac{\partial}{\partial x}\left(u_{x x}+2 u^{3}+6 u\right) \rightarrow \int_{-\infty}^{\infty} u d x=\text { const.(i), } \\
& \frac{\partial}{\partial t}\left(\frac{1}{2} u^{2}\right)=\frac{\partial}{\partial x}\left(\frac{3}{2} u^{4}+u u_{x x}-\frac{1}{2} u_{x}^{2}\right) \rightarrow \frac{1}{2} \int_{-\infty}^{\infty} u^{2} d x=\text { const.(ii), } \\
& \frac{\partial}{\partial t}\left(\frac{1}{4} u^{4}+u_{x}\right)=\frac{\partial}{\partial x}\left(u_{x x x}+6 u_{x}+6 u^{2} u_{x}\right) \rightarrow \int_{-\infty}^{\infty}\left(\frac{1}{4} u^{4}+u_{x}\right) d x=\text { const.(iii). }
\end{aligned}
$$

All conservation laws are proven by a direct calculation ${ }^{\circledR}$.

\section{Algebraic group properties (the classical case)}

In this section we use the classical Lie group analysis in order to derive new classes of solutions otherwise we are interested in the algebraic group behaviour of the nPDE (1).

Hint: In what follows we suppress the item 'classes'; so 'classes of solutions' are simply solutions.

We take up now the developments given in (Ibragimov, 1984; Olver, 1986; Bluman and Kumei, 1989; Gaeta, 1994; Huber, 2008; Huber, 2009) omitting all technical details. 
To use symmetry groups in any application, we first deduce the symmetries of eq.(1). The result is a system of eight linear homogeneous PDEs for the infinitesimals $\xi_{i}=\xi_{i}(x, u)$ and $\phi_{i}=\phi_{i}(x, u)$ :

$$
\begin{aligned}
& \frac{\partial \xi_{1}}{\partial u}=\frac{\partial \xi_{2}}{\partial u}=\frac{\partial \xi_{2}}{\partial x}=\frac{\partial^{2} \phi}{\partial u^{2}}=0, \\
& 12 u \phi-\frac{\partial \xi_{1}}{\partial t}-6 \frac{\partial \xi_{1}}{\partial x}-6 u^{2} \frac{\partial \xi_{1}}{\partial x}+6 \frac{\partial \xi_{2}}{\partial t}+6 u^{2} \frac{\partial \xi_{2}}{\partial t}-\frac{\partial^{3} \xi_{1}}{\partial t^{3}}+3 \frac{\partial \phi^{3}}{\partial x^{2} \partial u}=0, \\
& \frac{\partial \xi_{2}}{\partial t}-3 \frac{\partial \xi_{1}}{\partial x}=0, \\
& \frac{\partial^{2} \phi}{\partial x \partial u}-\frac{\partial^{2} \xi_{1}}{\partial x^{2}}=0, \\
& \frac{\partial \phi}{\partial t}+6 \frac{\partial \phi}{\partial x}+6 u^{2} \frac{\partial \phi}{\partial x}+\frac{\partial^{3} \phi}{\partial x^{3}}=0 .
\end{aligned}
$$

The infinitesimals are given by solving the above set of equations (3.1) to (3.5) leading to

$$
\begin{aligned}
& \xi_{1}=k_{2}+k_{3}(x+12 t) \\
& \xi_{2}=k_{1}+3 k_{3} t \\
& \phi=-k_{3} u(x, t)
\end{aligned}
$$

The result shows that the symmetry group of eq.(1) constitutes a finite three-dimensional point group containing translations in the independent variables and scaling transformations.

In (3.6) the group parameters are denoted by $k_{i}, i=1,2,3$. Eq.(1) admits the three-dimensional Lie algebra L of its classical infinitesimal point symmetries related to the following vector fields

$$
V_{1}=\partial_{t}, V_{2}=\partial_{x}, V_{3}=3 t \partial_{t}+(12 t+x) \partial_{x}-u \partial_{u} .
$$

These vector fields form a Lie algebra $\mathrm{L}$ by:

$$
\left[V_{1}, V_{3}\right]=-3 V_{1}+12 V_{2},\left[V_{2}, V_{3}\right]=V_{2},\left[V_{3}, V_{1}\right]=-3 V_{1}-12 V_{2},\left[V_{3}, V_{2}\right]=-V_{2} \text {. }
$$

For this three-dimensional Lie algebra the commutator table for $V_{i}$ is a $(3 \otimes 3)$ table whose $(i, j)$ th entry expresses the Lie Bracket $\left\lfloor V_{i}, V_{j}\right\rfloor$ given in (3.8). The table is skew-symmetric and the diagonal elements all vanish. The coefficient $C_{i, j, k}$ is the coefficient of $V_{i}$ of the $(i, j)$ th entry of Tab.1 and the related structure constants can be easily calculated from Tab. 1 to give:

$$
C_{1,3,1}=-3, C_{1,3,2}=-13, C_{2,3,2}=-1, C_{3,1,1}=3, C_{3,1,2}=12, C_{3,2,2}=1 .
$$

Tab.1 Commutator table for the Lie algebra $V$ of the nPDE (1).

\begin{tabular}{|c|c|c|c|}
\hline & $V_{1}$ & $V_{2}$ & $V_{3}$ \\
\hline$V_{1}$ & 0 & 0 & $-3 V_{1}-12 V_{2}$ \\
\hline$V_{2}$ & 0 & 0 & $-V_{2}$ \\
\hline$V_{3}$ & $3 V_{1}+12 V_{2}$ & $V_{2}$ & 0 \\
\hline
\end{tabular}

Theorem III: The Lie algebra of eq.(1) is solvable.

Proof: A Lie algebra $L$ is called solvable if $V^{(n)}=0$ for some $n>0 . V^{(1)}$ is an ideal containing $\left\{V_{1}, V_{2}, V_{3}\right\}, V^{(2)}$ is an ideal containing $\left\{V_{1}, V_{2}\right\}$; this can be reduced to $V^{(3)}=0$.

These subgroups are important later to perform a similarity reduction deducing new solutions.

The metric $(3 \otimes 3$ Cartanian tensor) satisfies: 


$$
g_{i j}=\left(\begin{array}{ccc}
0 & \cdots & 0 \\
\vdots & \ddots & \vdots \\
0 & \cdots & 12
\end{array}\right) \quad \text { with } \operatorname{det}(\mathrm{g})=0 .
$$

Since the condition $\operatorname{det}(g)=0$ holds the given algebra is degenerate.

Notes: Other useful algebraic group properties are worth to mention: Eq.(1) has no Casimir operator, the group order is three containing seven subgroups.

Alternatively, one can write eq.(3.10) with eq.(3.9) $g_{i m}=\sum_{i, k=1}^{n} c_{l k}^{i} c_{m i}^{k}$.

3.1 The derivation of similarity solutions

Let us now discuss the most important similarity solutions for special subgroups to derive new solutions where we restrict the analysis to the most important cases.

Case A: If we set the group parameters $k_{1}=1$ and $k_{3}=0$, the similarity variable and the relevant transformation reads as $x-\zeta=0$ and $S=u$. The related nODE of the third-order is

$$
\frac{d^{3} S}{d \zeta^{3}}+6 S^{2} \frac{d S}{d \zeta}+6 \frac{d S}{d \zeta}=0, \quad S \in \mathfrak{R}^{3}(-\infty, \infty), \quad \zeta \in(-\infty, \infty) .
$$

Case B: (the case of traveling waves): Here we assume the parameters to be $k_{1}=k_{2}=1$ and $k_{3}=0$ respectively. This choice means the traveling wave transformation by $t-x=\zeta$ and $S=u$.

The nODE of the third-order is calculated to

$$
\frac{d^{3} S}{d \zeta^{3}}+6 S^{2} \frac{d S}{d \zeta}+5 \frac{d S}{d \zeta}=0, \quad S \in \mathfrak{R}^{3}(-\infty, \infty), \zeta \in(-\infty, \infty)
$$

which differs from eq.(3.1.1) by a constant factor only Remark: For the given nODEs we both assume the existence of solutions and moreover, functions of the r.h.s. are continuously differentiable following a Lipschitz condition in the considered domain.

Also note that we enlarge the domain to ensure the possibility of complex-valued solutions.

\section{The non-classical case I - Potential symmetries}

For more technical details we refer to Ibragimov (1984), Olver (1986), Bluman and Kumei (1989), Gaeta (1994) and Huber (2009). For the nPDE (1) we found the following: The equation admits two possible potential systems $\Psi_{1}$ and $\Psi_{2}$.

Both systems can be formulated for two dependent variables $V_{i}, i=1,2$ and both variables are treated in their derivations w.r.t. the independent variables and are denoted by subscripts:

$$
\begin{aligned}
& \text { Potential system } \Psi_{1} \\
& 6 u+2 u^{3}-\frac{\partial V_{1}}{\partial t}+\frac{\partial^{2} u}{\partial x^{2}}=0 \\
& u+\frac{\partial V_{1}}{\partial x}=0
\end{aligned}
$$

\section{Potential system $\Psi_{2}$}

$$
\begin{aligned}
& 3 u^{2}+\frac{3 u^{4}}{2}-\frac{1}{2}\left(\frac{\partial u}{\partial x}\right)^{2}-\frac{\partial V_{2}}{\partial t}+u \frac{\partial^{2} u}{\partial x^{2}}=0 \\
& \frac{u^{2}}{2}+\frac{\partial V_{2}}{\partial x}=0
\end{aligned}
$$

The given systems are related to new symmetries which differ from the symmetry group (3.6) completely: In opposite to the symmetry group (3.6), here, we are confronted with a finite four- dimensional PT; that means that the difference exists in the dimension of the group as well as the number of the elements:

$$
\begin{aligned}
& \quad \text { Symmetry 1 } \\
& \begin{array}{l}
\xi_{1}=k_{3}+k_{4}(12 t+x) \\
\xi_{2}=k_{2}+3 k_{4} t \\
\phi_{1}=-k_{4} u \\
\phi_{2}=k_{1}
\end{array}
\end{aligned}
$$

$$
\begin{aligned}
& \text { Symmetry 2 } \\
& \xi_{1}=k_{3}+k_{4}(12 t+x) \\
& \xi_{2}=k_{2}+3 k_{4} t \\
& \phi_{1}=-k_{4} u \\
& \phi_{2}=k_{1}-k_{4} V_{2}
\end{aligned}
$$

If we examine the infinitesimals upon the dependence of variables starting with capital letter $V$, we realize that these infinitesimals are independent of the new potential variables.

Comparing this behavior with known results (e.g. the KdV Equation) we see that both systems do not contribute to potential symmetries (further examples are also well-known; e.g. the nonlinear Reaction Diffusion Equation, the cylindrical Korteweg de 
Vries Equation and the Burgers Equation) and tells us that potential symmetries are rare symmetries but can occur in connection with some equations.

\section{Approximate symmetries}

In this section we follow Huber (2009), Ibragimov (1985) and Ibragimov (1994), respectively and our intension is to deduce new results without referring too much theory. Let us introduce $\varepsilon$ as a small parameter $0<\varepsilon<<1$ determining the strength of the nonlinearity of eq.(1) so that we can write without loss of generality $\lambda=1$ :

$$
\frac{\partial u}{\partial t}+\frac{\partial^{3} u}{\partial x^{3}}-6 \varepsilon u^{2} \frac{\partial u}{\partial x}+6 \frac{\partial u}{\partial x}=0, \quad u \in \mathfrak{R}^{3}(-\infty, \infty),-\infty<x<\infty, t>0
$$

to ensure the complete solution-manifold. Then, approximate symmetries follow by

$$
\begin{aligned}
& \xi_{1}=k_{1}+4 k_{3} t+\frac{k_{3} x}{3}+\varepsilon\left(k_{5}+4 k_{7}+\frac{k_{7} x}{3}\right) \\
& \xi_{2}=k_{2}+k_{3} t+\varepsilon\left(k_{6}+k_{7}\right) \\
& \phi=-\frac{k_{3} u}{3}+\varepsilon\left(k_{8}+k_{4} u\right),
\end{aligned}
$$

representing an eight-dimensional approximate symmetry group in the first-order approximation.

The generating vector fields for this model read:

$$
\begin{aligned}
& V_{1}=\partial_{x}, \quad V_{2}=\partial_{t}, \quad V_{3}=t \partial_{t}+\left(-\frac{u}{3}\right) \partial_{u}+\left(4 t+\frac{x}{3}\right) \partial_{x}, \quad V_{4}=u \varepsilon \partial_{u}, \\
& V_{5}=\varepsilon \partial_{t}, \quad V_{6}=\varepsilon \partial_{u}, \quad V_{7}=\varepsilon t \partial_{t}+\left[\left(4 t+\frac{x}{3}\right) \varepsilon\right] \partial_{x}, \quad V_{8}=\varepsilon \partial_{x} .
\end{aligned}
$$

Possible reductions can be calculated by combining several sub-groups of (5.2). For the present calculations we choose three cases of interest:

Case A: Combining $V_{1} \otimes V_{2} \otimes V_{5}$ the transformation and the defining equation for $\mathrm{S}$ are:

$$
t-\frac{x}{1+\varepsilon}-\zeta=0, u=S, S^{\prime \prime \prime}+(1+\varepsilon)^{2}\left(5-\varepsilon+6 \varepsilon S^{2}\right) S^{\prime}=0, S=S(\zeta), S^{\prime}=d S / d \zeta .
$$

Case B: Combining $V_{1} \otimes V_{2} \otimes V_{6}$ one derives at:

$$
t-x(1-\varepsilon)-\zeta=0, u=S,(1-\varepsilon)^{3} S^{\prime \prime \prime}+\left(5+6 \varepsilon(1+\varepsilon) S^{2}\right) S^{\prime}=0 .
$$

Case C: Combining $V_{1} \otimes V_{5} \otimes V_{6}$ leads to the defining equation for $S(\zeta)$ :

$$
t-\frac{\varepsilon x}{1+\varepsilon}-\zeta=0, u=S, \varepsilon^{3} S^{\prime \prime \prime}+(1+\varepsilon)^{2}\left(-1+5 \varepsilon+6 \varepsilon^{2} S^{2}\right) S^{\prime}=0, \quad \varepsilon \neq 0 .
$$

The result is a similarity representation of the solution(s) linearly depending upon the perturbation parameter $\varepsilon$ and also in second and fourth-order dependence. Note that for the given nODEs the same assumptions as in Chapter 3.1 have been made.

\section{The non-classical case II: General symmetries (GS)}

We find it advisable mentioning some basic notes. It is obvious from Lie theory that point symmetries are a subset of generalized symmetries, Abramowitz and Stegun (1972) as well as Noether (1971) and Klein (1918). The determination of the characteristics for the general case follows by a similar algorithm as in the case of point transformations (PT) in the classical case.

Classical symmetries of a (n)PDE (assumed to be in a general form $\Delta=0$ ) are PT which guarantee the invariance of the solution space and so, PT are created by infinitesimal transformations.

The determining equations for the characteristics $G S_{\alpha}$ are consequences of the relation

$$
\left.\operatorname{pr} \vec{v}_{G S} \Delta\right|_{\Delta=0}=0 \text {, }
$$

where $\operatorname{pr} \overrightarrow{\mathrm{v}}_{G S}$ denotes prolongation of the vector field $\mathrm{v}_{G S}$ and 'GS' means generalize symmetry.

The main difference however is the fact that in general the characteristics depend upon derivatives of an infinite order. If the order is equal to identity we arrive at the so-called contact transformations. By increasing the order of derivatives $n>1$ we shall find higher order GS.

In case of eq.(1) we found GS of the first order depending on the first derivative:

$$
G S_{1}\left(x, t, u, u_{x}, u_{t}\right)=k_{1} u_{x} \text {. }
$$

This symmetry also changes from the symmetries given in (3.6), (4.3) and (5.1). Here we are confronted with a one- 
dimensional finite group of transformations where the second part $\partial u / \partial x$ is related to scaling and/or stretching operations (more precisely dilatations). For the case $n=2$ by assuming second partial derivatives we found

$$
\operatorname{GS}_{2}\left(x, t, u, u_{x}, u_{x x}, u_{t t}, u_{x t}\right)=k_{1} u_{x}
$$

as a quite similar result. At this stage we remark that higher cases are difficult to deal with.

\section{Analysis and results}

Now we use (3.1.1) and (3.1.2) respectively to derive new solutions. An analogues equation can be obtained if we investigate solutions for which $u=F(x, t), \quad F \in C^{3}(D), D \in R^{3}$ is a domain. Introducing the frame of reference $u(x, t)=U(\xi), \xi=x-\alpha t, \xi \in \Re^{1}, \alpha \in R \backslash\{0\}$ into eq.(1) leads to $U^{\prime \prime \prime}+6 U^{2} U^{\prime}+6 U^{\prime}-\alpha U^{\prime}=0$ and the prime means derivation w.r.t. $\xi$.

Due to the similar structure of the latter nODE it is sufficient to consider (3.1.1) and it can be shown that the traveling wave reduction results into eq.(3.1.2) exactly.

We summarize the analytical properties of eq.(3.1.1) resulting in a polynomial of the fourth-order in Tab.2. Thus we have to treat four cases depending upon the choice of the integration constants $K_{i}$ leading to new solutions:

For the Case A, $K_{1}=K_{2}=1$ appropriate numerical standard procedures are necessary.

Case B: $K_{1}=1$ and $K_{2}=0$ leads to a sine amplitude with a pure imaginary modulus:

$$
u(x)=-\left(11+\frac{21}{2^{2 / 3}}+102^{2 / 3}\right) s n\left[\sqrt{1+\frac{2\left(-4+2\left(2^{1 / 3}+2^{2 / 3}\right)\right) x}{2\left(2^{2 / 3}-22^{1 / 3}\right)-1+\sqrt{17-82^{2 / 3}}}, k}\right],
$$

with $k=\sqrt{\frac{1}{2}-1 / 2 \sqrt{1 / 573\left(7842^{1 / 3}+2602^{2 / 3}-307\right)}}$; that is numerically $k=0,4362 i$. The constant factor under the root sign is approximately $\approx 0,73$ and the first factor takes $\approx 2,5$.

Using a complex modulus transformation Abramowitz and Stegun (1972) we convert the function eq.(7) numerically into a realvalued function so that we have finally a pure local dependence

$$
u(x)=-2,3 \text { sd }[\sqrt{0,8 x}, k] \text { with the real modulus } k=0,9166 .
$$

A graphical plot for different values of the modulus shows Fig.1. In case of $k=1$ the function degenerates to the hyperbolic sine function as usual. For practical calculations a trigonometric series (Erdelyi, 1981), is sometimes useful so that one can write in a more convenient form:

$$
u(x)=\frac{4,6 \pi}{k k^{\prime} K} \sum_{n=1}^{\infty}(-1)^{n} \frac{q^{n-1 / 2}}{1+q^{2 n-1}} \sin (2 n-1) \frac{\pi \sqrt{0,8 x}}{2 K} \text { with the nome } q=\exp \left[-\pi\left(K^{\prime} / K\right),\right.
$$

valid in every strip of the form $|\operatorname{Im}(\pi x / 2 K)<1 / 2 \pi \operatorname{Im} \tau|$ and $K$ is the complete elliptic integral of the first kind.

Tab.2 Algebraic properties of the eq.(3.1.1) after converting. All zeros of the polynomial of the fourth-order $P(u)$.

The relating nODE of the first-order becomes $\int \frac{d u}{\sqrt{u^{4}+6 u^{2}+2 u+2}}=\left(\zeta-\zeta_{0}\right)$ and $\zeta_{0}$ is an arbitrary constant of integration.

\begin{tabular}{|c|c|c|c|}
\hline Case & $\begin{array}{c}\text { Integration } \\
\text { constants } K_{i}\end{array}$ & Polynomial $P(u)$ & Zeros $u_{i}$ of $P(u)$ \\
\hline A & $K_{1}=K_{2}=1$ & $u^{4}+6 u^{2}+2 u+2$ & $u_{1,2}=-0,18 \pm 0,56 i, u_{3,4}=0,18 \pm 2,40 i$ \\
\hline B & $K_{1}=1, K_{2}=0$ & $u^{4}+6 u^{2}+2 u$ & $u_{1}=0, u_{2}=2^{1 / 3}-2^{2 / 3}, u_{3,4}=\frac{-1 \pm 2 i \sqrt{3}+2^{1 / 3}(1 \pm i \sqrt{3})}{2^{2 / 3}}$ \\
\hline C & $K_{1}=0, K_{2}=1$ & $u^{4}+6 u^{2}+2$ & $u_{1,2}= \pm i \sqrt{3-\sqrt{7}}, u_{3,4}= \pm i \sqrt{3+\sqrt{7}}$ \\
\hline D & $K_{1}=K_{2}=0$ & $u^{4}+6 u^{2}$ & $u_{1,2}=0, u_{3,4}= \pm i \sqrt{6}$ \\
\hline
\end{tabular}


A formal power expansion up to order three yields

$$
u(x)=2,28+1,36(x-1)-0,18(x-1)^{2}+0,11(x-1)^{3}+O[x-1]^{4} .
$$
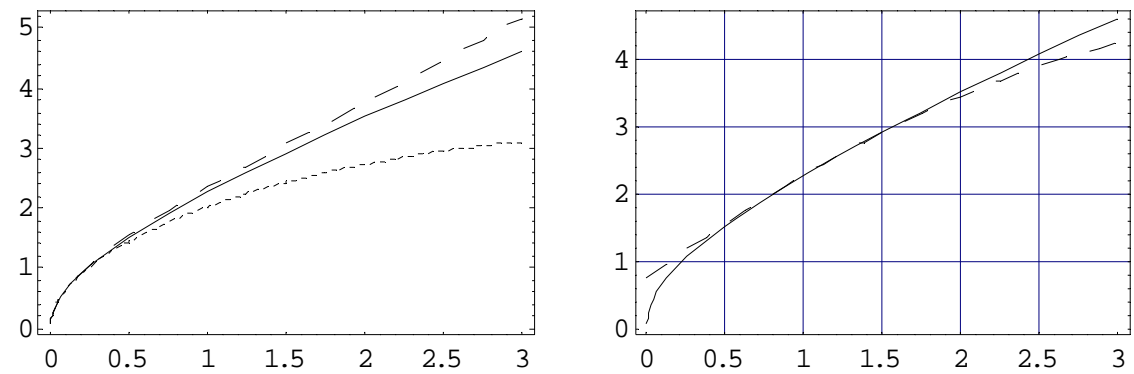

Fig.1 Left: Some solution curves of the function (7) for different values of the modulus: $k=0,9166$ (solid line), $k=0,5$ (short dotted line), $k=1$ (dotted line) - this case degenerates to the sinh function. Right: The undisturbed solution eq.(7.1), (solid line) by comparison with the second-order approximation calculated from the power expansion (7.3) respectively, (dotted line).

This can be compared with the exact solution in Fig.2 and therefore we conclude that the expansion is valid in the domain $0,3<x<2$. One can also make use of the Weierstrassian expansion [14].

Case $C: K_{1}=0$ and $K_{2}=1$ leads to complex-valued solutions for $x \in \subseteq^{+}, x>0$ :

$$
u(x)=-\frac{i}{4}\left\{A s n\left[i \sqrt{\frac{(\sqrt{17}-5) x}{2}}, k\right]\right\} \text { with } A=\left\{\frac{20}{\sqrt{95+23 \sqrt{17}}}+4 \sqrt{\frac{17}{95+23 \sqrt{17}}}\right\} .
$$

Note that the modulus is $k>1$, that is $k=(21 / 4+5 \sqrt{17} / 4)^{1 / 2}=3,2255$.

To proceed further one has to use suitable transformations so that $0<k<1$ holds. Refering to [19] we calculate

$$
u(x)=A \operatorname{tn}\left[\sqrt{\frac{(\sqrt{17-5) x}}{2}}, k^{\prime}\right],
$$

where the new modulus $k^{\prime}$ is given by $k^{\prime}=\sqrt{1-k^{2}}=0,9954$.

We also note that for $x<1, x \neq 0$ the function (6.6) takes real value. The development of this function in the complex plane shows Fig. 2 by using different initial values and Fig. 3 shows the real-valued function considering the assumption $x<1$.
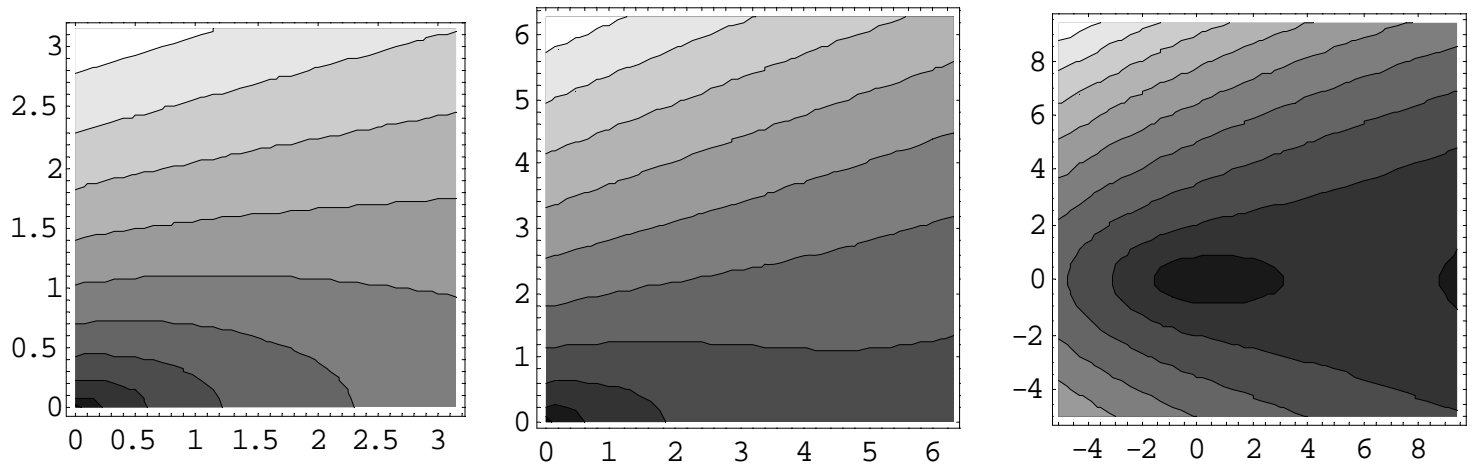

Fig.2. The behavior of the branch lines near a zero of the function (7.5) in the complex plane with $A \approx 2$. Different values for the complete integral of the first kind are used: Right: The complete integral is assumed to $K=2$, middle: $K=4$ and left: $K=6$. 


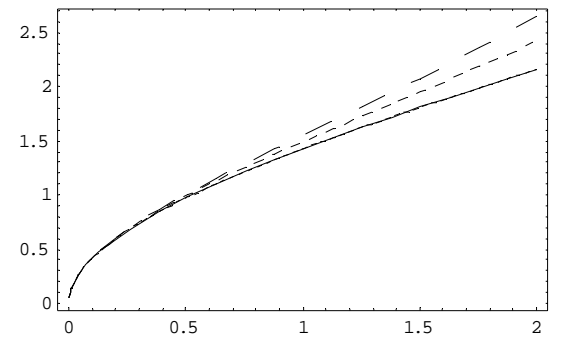

Fig.3 The real-valued tn-function for different values for the modulus and $A \approx 2$; solid line: $k^{\prime}=0,9954$, dotted line: $k^{\prime}=0,5$ and short dotted line: $k^{\prime}=1$. Note: The tn-function degenerates to the sinh function.

Case D: $K_{1}=K_{2}=0$ :

$$
u(x)=\frac{12 e^{\sqrt{6}(1+x)}}{e^{2 \sqrt{6}}-6 e^{2 \sqrt{6} x}}=\frac{12(\operatorname{Cosh}(\sqrt{6}(1+x))+\sinh (\sqrt{6}(1+x)))}{c+6(\sinh (2 \sqrt{6} x)-\cosh (2 \sqrt{6} x))}
$$

where $c$ means $(\sinh (2 \sqrt{6})-\cosh (2 \sqrt{6}))$ and the constant $x_{0}$ is assumed to be the identity. For this solution we calculate a closed-form analytical expression in terms of infinite series

$$
u(x)=\frac{\sum_{k=0}^{\infty} \frac{2^{2+k / 2} 3^{1+k / 2} e^{\sqrt{6}}}{k !} x^{k}}{c+\sum_{k=0}^{\infty} \frac{2^{2+3 k / 2} 3^{1+k / 2} e^{\sqrt{6}}}{k !} x^{k}},
$$

where the convergence $\forall x \in \subseteq$ can be proven by using the d'Alembertian criterion immediately. For large values of the argument, say $|x| \rightarrow \infty$ the following asymptotic formula holds:

$$
u(x) \sim \frac{12 e^{\sqrt{6}}}{6+c}+\frac{12 \sqrt{6}(c-6)}{(6+c)^{2}}\left(\frac{1}{x}\right)+\frac{36\left(36+(c-36) c e^{\sqrt{6}}\right.}{(6+c)^{3}}\left(\frac{1}{x}\right)^{2}+O\left(\frac{1}{x}\right)^{3} .
$$

It is proven that the following limiting behaviour holds: $\lim u(x) \approx 1$ and $\lim u(x)=0$; the first and the second derivation are finite at the point $x=0$.

It is remarkable that the analysis of eq.(3.1.2) also leads to similar results depending upon the choice of the integration constants. Although eq.(3.1.2) admits the case of traveling motion which is concern to the appropriate similarity variable and no classical wave propagation is observed.

Finally, we discuss the equations relating to approximate symmetries eq.(5.3), (5.4) and (5.5).

The first and the second equations lead to a linear ODE of the third-order by setting $\varepsilon=0$, that is $S^{\prime \prime \prime}+5 S^{\prime}=0$ and the prime means derivation w.r.t. $\zeta$.

An analytical solution of the first-order approximation is therefore:

$$
S(\zeta)=\frac{1}{\sqrt{5}}\left\{c_{1} \cos \sqrt{5} \zeta+c_{2} \sin \sqrt{5} \zeta\right\}+c_{3}+O[\varepsilon],
$$

where the $c_{i}$ are arbitrary constants. This case covers the traveling wave solution for $t-x /(1+\varepsilon)-\zeta=0$ if we set $\varepsilon=0$. For $\zeta \rightarrow 0$ the function(s) takes a finite value but remains indefinite as $\zeta \rightarrow \pm \infty$. For solution eq.(6.5) a closed-form analytical expression can be obtained:

$$
S(\zeta)=\sum_{k=0}^{\infty} \zeta^{2 k}\left[\frac{(-1)^{k} 5^{-\frac{1}{2}+k}}{(2 k) !}+\frac{(-5)^{k}}{\Gamma(2+2 k)}\right],
$$

where the convergence is also proven immediately. A graphical overview for the traveling motion represents Fig.4.

To analyze eq.(5.5) we expand about the fourth-order term by setting $\varepsilon=1$ and a solution of the fourth-order approximation is given:

$$
S(\zeta)=-1+2 \varepsilon+\left(11+6 \zeta^{2}\right) \varepsilon^{2}+\left(16+30 \zeta^{2}\right) \varepsilon^{3}+O[\varepsilon]^{4}
$$


leading to a quadratic dependence. We show some solution curves by using different initial conditions in Fig.4. Here, the traveling motion as well as the quadratic dependence is remarkable.
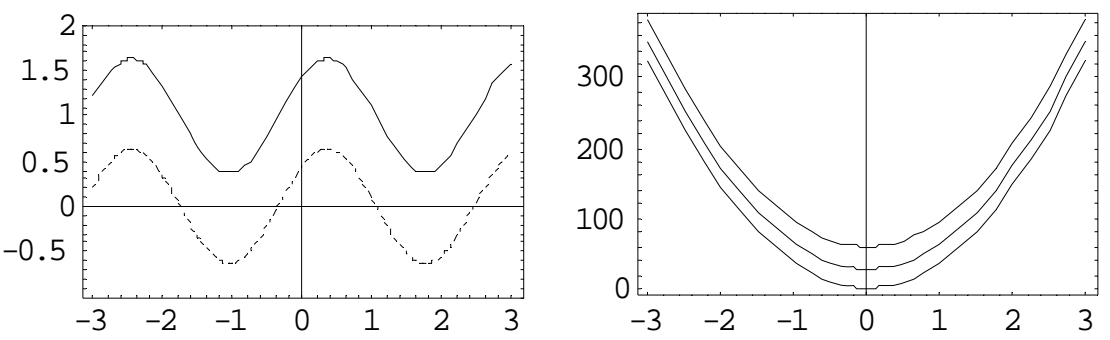

Fig.4 Left: Solutions of eq.(7.9) representing traveling waves by using different values of the integration constant $c_{i}$, especially $c_{1}=c_{2}=1$ and $c_{3}=1$ (solid line), $c_{1}=c_{2}=1, c_{3}=0$ (dotted line); the perturbation parameter is assumed to be $\varepsilon=0$. Right: The quadratic dependence of eq.(7.11) in fourth-order approximation.

\section{Conclusion remarks - main propositions - outlook}

In this paper a less studied nPDE of the third-order is under consideration.

Let us emphasise in brief the results of the analysis. Usually by introducing special similarity variables, say $u(x, t)=U(\xi)$ with $\xi=x-\alpha t$, one would expect traveling motion as a result. For this special nPDE we did not found such solutions in the general case.

However, by choosing suitable values of the constants involved the traveling behaviour results. In addition the nPDE admits conservation laws derived for the first time similar to the $\mathrm{KdV}$ and analogues equations. The derived conservation laws are connected directly with physical measurable quantities like mass, the horizontal momentum and the energy. The dispersion relation, the group and the phase velocity as further physically important quantities are in agreement with many other evolution equations. It is important to point out that we apply a classical group analysis to generate new solutions for the first time. The nonclassical case, also performed for the first time leads to the expected traveling wave result. A further important contribution shows that the $\mathrm{nPDE}(1)$ does not allow potential symmetries (similar to the $\mathrm{KdV}$ ).

It is known that similarity 'ansätze' of the form $\xi=x-\alpha t$ does not guarantee the existence of physically important wave propagation; the nPDE (1) is a notable example for this behavior. We also show the existence of approximate and generalized symmetries to the first time.Finally, it is seen that the nPDE(1) does not belong to the hierarchy of the KdV Equation. Naturally, the next step is to prove the integrability by assuming that the $\mathrm{nPDE}(1)$ can be written in form of an equation of motion, the socalled Lax equation $u_{t}=[L, B]$, where $L$ means the Schrödinger operator (as a first assumption) which commutate with the commutator $B$. If so, we then can show that the $\mathrm{nPDE}(1)$ is integrable completely possessing infinitely many laws of conservation and has a related Bäcklund system.

\section{References}

Drazin P., Johnson R., 1989. Solitons: An Introduction, Cambridge University Press.

Witham G., 1974. Linear and Nonlinear Waves, Wiley, N.Y, p.577.

Eilenberger G., 1983. Solitons, Springer Verlag, Berlin, p.140.

Ablowitz M., Clarkson P., 1991. Solitons, Nonlinear Evolution Equations and Inverse Scattering, Cambridge University Press.

Dodd R., Eilbeck J., Gibbon J., Morris H., 1988. Solitons and Nonlinear Wave Equations, Academic Press.

Zabusky N., 1967. A synergetic approach to problems of nonlinear dispersive wave propagation and interaction, Proc. Symp. on Nonlinear Partial Differential Equations (ed. by W. Ames), Academic Press, Boston, p.223.

Kakutani T., Ono H., 1969. Weak nonlinear hydromagnetic waves in a cold collision-free plasma, J. Phys. Soc. Jpn. 31, p.1246.

Fung P. C. W., Au C., 1984. A series of a new analytical solution to the nonlinear equation $y_{t}+y_{x x x}+6 y^{2} y_{x}+6 \lambda y_{x}=0$, J. Math.

Phys. Vol. 25, No. 5, p.1370.

Au C., Fung P. C. W., 1984. A KdV soliton propagating with varying velocity, J. Math. Phys. 25, p.1364.

Ibragimov N., 1994. Lie Group Analysis, Vol. III, CRC Press, Inc.

Olver P., 1986. Applications of Lie Groups to Differential Equations, Springer.

Bluman G., Kumei S., 1989. Symmetries and Differential Equations, Springer

Gaeta G., 1994. Nonlinear Symmetries and Nonlinear Equations, Kluwer, Acad. Press.

Huber A., 2008. A note on new solitary and similarity class of solutions of a fourth order nonlinear evolution equation, Appl. Math. and Comp., Vol. 202, p.787 
Huber A., 2009. The Cavalcante-Tenenblat equation - Does the equation admit a physical significance? Appl. Math. and Comp. 212, p.14.

Ibragimov N., 1985. Transformation Groups Applied to Mathematical Physics, Reidel Publ., Dortrecht. Ibragimov N., 1994. Sophus Lie and harmony in mathematical physics on the $150^{\text {th }}$ anniversary of his birth, Math. Intel. 16, p.20. Erdelyi A., 1981. Higher Transcendent Functions, N. Y., Krieger.

Abramowitz M., Stegun I., 1972. Handbook of Mathematical Functions, Tenth Printing.

Noether E., 1971. Transport Theory Stat. Phys. Vol. 1, p.186.

Klein F., 1918. Über Differentialgesetzte für die Erhaltung von Impuls und Energie in der Einsteinschen Gravitationstheorie, Nachr. Ges. Wiss. Göttingen Math. Phys.2, p.171.

\section{Biographical notes}

Dipl.-Ing. Dr. techn. Huber Alfred is a distinguished lecturer at the Institute of Theoretical Physics - Computational Physics at the Technical University Graz, Austria following his habilitation treatise. He did his diploma thesis titled 'Systematic in the physics of elementary particles focusing the quarkonium states' in the field of elementary particle physics at the former Institute of Nuclear Physics at the Technical University Graz, Austria. He completed his scientific education with the doctoral programme of technical sciences at the Institute of Chemical Technology of Inorganic Compounds at the Technical University Graz, Austria subject to nuclear solid state physics and advanced electrochemistry. Thesis titled 'Synthesis and characterization of doped $\gamma$-manganese dioxides'. Also the author has a learnt vocation for a chemical assistant at the Research Centre of Electron Microscopy at the former Technical High School Graz, Austria. He is the author of 27 articles which have appeared in world-wide renowned scientific journals. His research interests are nonlinear partial differential equations (nPDE) of higher order with applications especially in physics and chemistry. The author developed several new algebraic procedures for solving nPDE. Special interests are further given in classical and non-classical symmetry methods, nonlinear transformations and the application of nonlinear methods in describing electrochemical interfaces, nonlinear wave propagation and further nonlinear topics of advanced character.

Received March 2010

Accepted April 2010

Final acceptance in revised form April 2010 